\title{
JOINT CONTINUITY \\ OF DIVISION OF SMOOTH FUNCTIONS. II: THE DISTANCE TO A WHITNEY STRATIFIED SET FROM A TRANSVERSAL SUBMANIFOLD
}

\author{
BY
}

\author{
MARK ALAN MOSTOW
}

\begin{abstract}
Let $S$ be a closed set in $\mathbf{R}^{m}$, and let a $C^{1}$ Whitney stratification of $S$ be given. (Actually, only Whitney (a)-regularity is needed.) Let $f: \mathbf{R}^{n} \rightarrow \mathbf{R}^{m}$ be a $C^{1}$ map transversal to all the strata. Assume that the image of $f$ intersects $S$. Then for each compact set $K$ in $\mathbf{R}^{n}$, the Euclidean distances $\rho\left(x, f^{-1}(S)\right)$ and $\rho(f(x), S)$, for $x$ in $K$, are bounded by constant multiples of each other. The bounding constants can be chosen to work for all maps $g$ which are close enough to $f$ in a $C^{1}$ sense on a neighborhood of $K$. This result is used in part I (written jointly with S. Shnider) to prove a result on the joint continuity of the division of smooth functions [MS].
\end{abstract}

Introduction. In the course of studying quotients of smooth functions, one is led to consider properties of the zero sets of the denominators. In his proof that division of distributions by an analytic function is possible, Lojasiewicz [Loj] studied certain stratifications of the zero set of an analytic function. He proved that the strata satisfy a certain metric condition which he called regular separation. Malgrange [Mal, p. 14] defined an equivalent condition on the closures of the strata. In his terminology, one says that two closed sets $X, Y \subset U$, for $U$ an open set in $\mathbf{R}^{m}$, are regularly situated if either

(1) $X \cap Y$ is empty, or

(2) given any pair of compact sets $K \subset X, L \subset Y$, there exists a pair of positive constants $C$ and $\mu$ such that for every $x$ in $K$, one has

$$
\rho(x, L) \geqslant C(\rho(x, X \cap Y))^{\mu}
$$

(here $\rho$ denotes Euclidean distance in $\mathbf{R}^{m}$ ). This condition is symmetric in $X$ and $Y$, using the same exponent $\mu$ locally ([Loj, p. 91]; see also Lemma 3 below). One gets an equivalent definition if $\rho(x, L)$ is replaced by $\rho(x, Y)$. The exponent $\mu$ can always be increased. It is of necessity $\geqslant 1$ if $K$ intersects $L$ (respectively $Y$ ), and can be chosen to equal any positive number otherwise. Lojasiewicz showed [Loj; Mal, p. 62 , Corollary 4.4] that any two analytic subsets of $\mathbf{R}^{n}$ are regularly situated and that the zero set of an analytic function admits a stratification for which the closures of the strata are regularly situated. From this fact, together with growth conditions for an analytic function near its zero set, Lojasiewicz was able to deduce his division

Received by the editors December 31, 1984.

1980 Mathematics Subject Classification. Primary 46E25; Secondary 26E10, 46E10, 46H10.

Key words and phrases. Whitney stratification, transversality, Whitney (a)-regularity, regular separation. 
theorem. One easily sees, however, that none of the stratifications of the zero set of $g(x, y)=y\left(y-\exp \left(-1 / x^{2}\right)\right)$ has the property of regular situation, even though $g$, too, generates a closed ideal and thus division of a distribution by $g$ is defined (cf. [MS]).

While investigating the joint continuity of division of smooth functions [MS], the author and Steve Shnider encountered a similar problem. We needed to show that given a closed Whitney stratified set $S \subset \mathbf{R}^{m}$ and a closed $C^{1}$ submanifold $N \subset \mathbf{R}^{m}$ which is transversal to all the strata of $S, N$ and $S$ are regularly situated. We also needed to prove that the constants $C$ and $\mu$ can be chosen to work even when $N$ is moved a bit, in a $C^{1}$ sense. The main result of this paper is that such constants can be found. In fact, the exponents $\mu$ can all be taken to be 1 . Moreover, the result holds without any analyticity or metric conditions on the stratified set. It is valid, in particular, even for the set $g^{-1}(0)$ discussed above. The stratification needs only to satisfy Whitney's (a)-regularity condition.

It is instructive to prove the result first in the special case that $S$ is also a submanifold. The problem reduces to local problems around each point of $N \cap S$. For each local problem, transversality and the Inverse Function Theorem [GG] imply that we can apply a $C^{1}$ diffeomorphism which flattens out $S$ and $N$ simultaneously to linear subspaces of $\mathbf{R}^{m}$. If we assume, as we may, that these subspaces are coordinate planes (possibly of different dimensions), the result is obvious since $\rho(x, N)$ then equals $\rho(x, N \cap S)$ for all $x \in S$. To facilitate comparison with the argument when $S$ is a stratified set, however, we shall not make this assumption. Then for any point $x \in S$ the ratio $\rho(x, N) / \rho(x, N \cap S)$ is the sine of the angle formed by a certain pair of lines, one lying in $N$ and one in $S$, both perpendicular to $N \cap S$. By a compactness argument these angles have a positive lower bound, and so, too, do their sines.

In the general case we can flatten $N$ but not $S$ locally. Moreover, we must take into account that the points of $S$ and of $N \cap S$ closest to a given point $x \in N$ may lie in different strata. Nonetheless, it turns out to be possible to adapt the angle proof to the general case. Whitney (a)-regularity is precisely the condition we need, since it requires that the tangent spaces of the different strata match up well, as a result of which the stratified set $S$ behaves in some ways like a submanifold.

\section{Definitions and precise statements of results.}

Definitions (Following [Tro AND GG]). A stratification $\Sigma$ of a subset $S$ of a $C^{1}$ manifold $M$ is a partition of $S$ into (disjoint) connected $C^{1}$ submanifolds of $M$, called the strata of $\Sigma$, in a locally finite way, meaning that each point of $S$ has a neighborhood meeting only finitely many strata. The pair $(S, \Sigma)$, or by abuse of terminology the set $S$, is called a stratified set. It is (a)-regular (in the sense of Whitney) if for each pair of strata $A, B$ of $\Sigma, B$ is (a)-regular over $A$, meaning that for each point $x \in A \cap \mathrm{Cl}(B)$,

If $\left\{x_{j}\right\}$ is a sequence of points in $B$ tending to $x$, such that the tangent spaces $T_{x_{j}} B$ tend to a linear subspace $T \subset T_{x} M$, then $T_{x} A \subset T$. 
A Whitney stratification is a stratification which satisfies Whitney (b)-regularity [Wh2], a condition stronger than (a)-regularity. Our results will be valid for (a)regular stratifications, and hence, in particular, for Whitney stratifications.

A $C^{1}$ map $f: N \rightarrow M$ between $C^{1}$ manifolds is transversal to a $C^{1}$ submanifold $W \subset M$ if $d f\left(T_{x} N\right)+T_{f(x)} W=T_{f(x)} M$ for all $x \in f^{-1}(W)$. The map $f$ is transversal to a stratified set $(S, \Sigma)$, where $S \subset M$, if $f$ is transversal to $A$ for all strata $A$. Two $C^{1}$ submanifolds $V, W \subset M$ are transversal, or intersect transversally, if $T_{x} V+T_{x} W=$ $T_{x} M$ for all points $x \in V \cap W$. A $C^{1}$ submanifold $V$ is transverisal to a stratified set $(S, \Sigma)$ if it is transversal to every stratum.

THEOREM. Let $S$ be a closed subset of $\mathbf{R}^{m}$, and let $\Sigma$ be an (a)-regular $\left(C^{1}\right)$ stratification of $S$ (for example, a Whitney stratification). Let $N$ be a closed embedded $C^{1}$ submanifold of $\mathbf{R}^{m}$ which is transversal to $(S, \Sigma)$. Then $N$ and $S$ are regularly situated with exponent $\mu=1$. That is, either $N \cap S$ is empty, or it is not empty and for each compact subset $K$ of $S$ one can find a positive constant $C$ so that

$$
\rho(z, N) \geqslant C \rho(z, N \cap S) \text { for all } z \in K .
$$

Equivalently, if $L$ is compact in $N$, there exists $C^{\prime}>0$ such that

$$
\rho(y, S) \geqslant C^{\prime} \rho(y, N \cap S) \text { for all } y \in L .
$$

Here $\rho=\rho_{m}(\cdot, \cdot)$ is the standard Euclidean distance function on $\mathbf{R}^{m}$.

Corollary 1. Let $S, \Sigma$ and $\rho$ be as in the Theorem. Let $f: \mathbf{R}^{n} \rightarrow \mathbf{R}^{m}$ be a $C^{1}$ map transversal to $(S, \Sigma)$. Assume that $f^{-1}(S)$ is not empty. Let $K$ be a compact set in $\mathbf{R}^{n}$. Then the distances $\rho_{n}\left(x, f^{-1}(S)\right)$ and $\rho_{m}(f(x), S)$, for $x \in K$, are bounded by constant multiples of each other.

COROLlary 2. Assume the situation of Corollary 1. Let $U$ be an open neighborhood of $K$. Then there exists a constant $\delta>0$ such that the bounding constants in Corollary 1 can be chosen to work for all $C^{1}$ maps $g: \mathbf{R}^{n} \rightarrow \mathbf{R}^{m}$ satisfying $|g-f|<\delta$ and $\left|\partial(g-f) / \partial x_{i}\right|<\delta$ on $U$ for all $i=1, \ldots, n$.

REMARK 1. The Theorem becomes false if "closed" is replaced by "locally closed" in the hypotheses on $S$ or on $N$. It fails, for example, if $S$ is the complement in $\mathbf{R}^{2}$ of a closed interval on the $x$-axis and $N$ is the $x$-axis.

REMARK 2. The Theorem and corollaries can be extended to $C^{1}$ stratifications of arbitrary $C^{1}$ manifolds, using the distance function associated to any (continuous) Riemannian metric. To see this, observe that (1) the problems are local, and (2) any two Riemannian metrics define distance functions which locally are bounded by constant multiples of each other.

2. The angle between affine subspaces. In this section we develop a notion of the angle between two affine subspaces of Euclidean space. The results proved here will be used to make rigorous the proof of the special case sketched in the Introduction and to adapt that proof to the general case.

LEMMA 1. Let $E, F$ be affine subspaces of $\mathbf{R}^{m}$ whose respective underlying vector spaces $H, K$ are transversal (i.e. $H+K=\mathbf{R}^{m}$ ). Then $E \cap F$ is not empty. 
REMARK. Trivially, $E$ and $F$ are transversal as submanifolds.

Proof of Lemma 1. Suppose $E \cap F$ is empty. Pick points $e \in E, f \in F$ at minimal distance from each other. They determine a line ef which is perpendicular to $E$ and to $F$, hence to $H+K=\mathbf{R}^{m}$. But this is impossible. Q.E.D.

DEFINITION. If $E, F$ are affine subspaces of $\mathbf{R}^{m}$ (with its standard Euclidean metric), define the angle $\theta(E, F)$ between them to be the infimum of $\theta(l, m)$ over all pairs $(l, m)$, where $l \subset E, m \subset F$ are intersecting lines perpendicular to $E \cap F$ and $\theta(l, m)$ is the acute or right angle between them. If the set of such pairs $(l, m)$ is empty, define $\theta(E, F)$ to be 0 .

REMARK. The set of pairs $(l, m)$ is empty if and only if $E \cap F$ is empty or $E \subset F$ or $F \subset E$.

Comparison with Kuo's distance function. If $E$ and $F$ intersect, the angle $\theta(E, F)$ can be thought of as the smallest angle through which $E$ or $F$ can be rotated to decrease the dimension of $H+K$, or equivalently, to increase the dimension of $E \cap F$. In contrast, the distance $\delta(H, K)$ between two linear subspaces $H, K$ in $\mathbf{R}^{m}$, as defined by Kuo [Kuo], is the sine of the supremum, over all lines $l$ through 0 in $H$, of the acute or right angle between $l$ and its orthogonal projection in $K$. Our angle function $\theta$ is always symmetric in its two arguments; Kuo's distance function $\delta$ is not unless the two subspaces have the same dimension. On the other hand, $\delta$ is continuous in its arguments, while $\theta$ is not, as we are about to show.

Proposition. The angle $\theta(E, F)$ between two affine subspaces $E, F$ of $\mathbf{R}^{m}$ has the following properties:

(a) $\theta(E, F)$ is symmetric in $E$ and $F$.

(b) $0 \leqslant \theta(E, F) \leqslant \pi / 2$.

(c) If $E, F$ are affine subspaces of $\mathbf{R}^{m}$ with nonempty intersection, and if $H, K$ are their respective underlying linear subspaces, then $\theta(E, F)=\theta(H, K)$. In particular, equality always holds if $H$ and $K$ intersect transversally.

(d) $\theta(E, F)=0$ if and only if $E \subset F$ or $F \subset E$ or $E \cap F$ is empty.

(e) If $\theta(E, F) \neq 0$, there exist lines $l \subset E, m \subset F$, both perpendicular to $E \cap F$, with $\theta(E, F)=\theta(l, m)$.

(f) $\theta(H, K)$ is not continuous in $(H, K)$, in general, as $H$ ranges over the Grassmannian of h-dimensional linear subspaces of $\mathbf{R}^{m}$ and $K$ ranges over the Grassmannian of $k$-dimensional linear subspaces. It is continuous, however, on any subspace of pairs $(H, K)$ for which $\operatorname{dim}(H \cap K)$, or equivalently $\operatorname{dim}(H+K)$, does not vary.

(g) If $H_{j}, K_{j}$ are sequences of linear subspaces of $\mathbf{R}^{m}$ of dimension $h<m$, resp. $k<m$, which satisfy $H_{j} \rightarrow H, K_{j} \rightarrow K$ (in the respective Grassmannians), $H_{j}+K_{j}=$ $\mathbf{R}^{m}$ for all $j$, and $\theta\left(H_{j}, K_{j}\right) \rightarrow 0$ as $j \rightarrow \infty$, then $H+K \neq \mathbf{R}^{m}$. It need not be the case, however, that $\theta(H, K)=0$.

Proof of Proposition. (a), (b), and (c) are obvious (using Lemma 1 for the second part of (c)). The "if" part of (d) follows from the definition of $\theta(\cdot, \cdot)$. For the "only if" part of (d) and for (e), assume that $E \cap F$ is not empty, $E \not \subset F$ and $F \not \subset E$. By (c), we may reduce to the case of linear subspaces $E=H$ and $F=K$. Using translation we may restrict attention to lines $l, m$ which pass through 0 . Since the 
projective spaces of lines through the origin in $H \cap(H \cap K)^{\perp}$, resp. $K \cap$ $(H \cap K)^{\perp}$, are compact and not empty, and since $\theta(l, m)$ is a continuous function of $(l, m)$, the infimum $\inf (\theta(l, m))$ defining $\theta(H, K)$ is attained, say by lines $l^{\prime}, m^{\prime}$. If $\theta(H, K)$ were zero, the lines $l^{\prime}$ and $m^{\prime}$ would be the same and would lie in $H$, in $K$ and in $(H \cap K)^{\perp}$ simultaneously, which is impossible. Hence $\theta(H, K) \neq 0$.

A counterexample to the continuity of $\theta(H, K)$ is given by $m=4, H=$ $\operatorname{span}\left(e_{1}, e_{2}\right), K_{t}=\operatorname{span}\left(e_{1}+t e_{3}, \dot{e}_{4}\right)$, where $\left\{e_{j}\right\}, j=1, \ldots, 4$, is the standard basis of $\mathbf{R}^{4}$ and $t$ is a real parameter. When $t=0, H \cap K_{t}=\operatorname{span}\left(e_{1}\right)$ and $\theta\left(H, K_{0}\right)=$ $\theta\left(\operatorname{span} e_{2}, \operatorname{span} e_{4}\right)=\pi / 2$. When $t \neq 0, H \cap K_{t}=0$ and $\theta\left(H, K_{t}\right)=\theta\left(e_{1}, e_{1}+t e_{3}\right)$ $=\tan ^{-1}|t|$. As $t \rightarrow 0, K_{t} \rightarrow K_{0}$, but

$$
\theta\left(H, K_{t}\right) \rightarrow 0 \neq \pi / 2=\theta\left(H, K_{0}\right) .
$$

This proves the first half of (f). The second part of (f) follows easily from the observation that if $H_{j} \rightarrow H, K_{j} \rightarrow K$ and $\operatorname{dim}\left(H_{j} \cap K_{j}\right)=\operatorname{dim}(H \cap K)=d$ [or equivalently $\left.\operatorname{dim}\left(H_{j}+K_{j}\right)=\operatorname{dim}(H+K)\right]$ for all $j$, then $H_{j} \cap K_{j} \rightarrow H \cap K$ in the Grassmannian of $d$-planes through the origin in $\mathbf{R}^{m}$.

The first part of (g) follows from (d) and the second part of ( $\mathrm{f}$ ). The example given for ( $\mathrm{f}$ ) establishes the second part of (g). Q.E.D.

3. Proofs of the Theorem and corollaries. If $A$ is a submanifold of $\mathbf{R}^{m}$ and $x \in A$, we can regard $T_{x} A$ either as a linear subspace of $T_{x} \mathbf{R}^{m} \approx \mathbf{R}^{m}$ or as an affine subspace of $\mathbf{R}^{m}$ containing the point $x$.

Lemma 2. Let $A, B \subset \mathbf{R}^{m}$ be $C^{1}$ submanifolds, with $B$ Whitney (a)-regular over $A$. Let $N \subset \mathbf{R}^{m}$ be a $C^{1}$ submanifold transversal to $A$ at a point $x \in N \cap A$. Then:

(1) There exists a neighborhood $U$ of $x$ in $\mathbf{R}^{m}$ such that for all $z \in U \cap B, T_{x} B$ and $T_{x} N$ are transversal as linear subspaces of $\mathbf{R}^{m}$ (or equivalently as affine subspaces).

(2) If $\operatorname{dim} B<m$ and $\operatorname{dim} N<m$, there exist a neighborhood $U$ of $x$ in $\mathbf{R}^{m}$ and a positive angle $\theta_{0}$ such that for all $z \in U \cap B, \theta\left(T_{x} B, T_{x} N\right) \geqslant \theta_{0}$.

Proof of Lemma 2. If (1) were false, we could find a sequence $\left\{z_{j} \in B\right\}$ converging to $x$ for which $T_{z_{j}} B+T_{x} N \neq \mathbf{R}^{m}$ for all $j$ (as linear subspaces of $\mathbf{R}^{m}$ ). Since the Grassmannian of $b$-planes of $\mathbf{R}^{m}(b=\operatorname{dim} B)$ is compact, we may replace $\left\{z_{j}\right\}$ by a subsequence so that $T_{z_{j}} B$ converges to a $b$-plane $T \subset \mathbf{R}^{m}$. By Whitney (a)-regularity, $T_{x} A \subset T$. Since $T_{x} A+T_{x} N=\mathbf{R}^{m}$ by hypothesis, $T$ is transversal to $T_{x} N$. But transversality to $T_{x} N$ is an open condition in the Grassmannian, hence is satisfied by all $T_{z_{j}} B$ for large enough $j$, contrary to supposition.

Similarly, if (2) were false we could find another sequence $\left\{w_{j}\right\}$ in $B$ converging to $x$, with $\theta\left(T_{w_{j}} B, T_{x} N\right) \rightarrow 0$ and $T_{w_{j}} \rightarrow T^{\prime}$. Since (1) is true, we may arrange that $T_{w_{j}} B+T_{x} N=\mathbf{R}^{m}$ for all $j$. By part (g) of the Proposition, $T^{\prime}+T_{x} N \neq \mathbf{R}^{m}$. But this is false by the argument used before. Q.E.D.

Lemma 3. Let $N$ and $S$ be any closed subsets of $\mathbf{R}^{m}$, with $N \cap S$ not empty, and assume that $x \in S$. Let $U$ be a neighborhood of $x$ in $\mathbf{R}^{m}$, and let $V$ be the closed ball of radius $r$ about $x$. Assume that a closed ball of radius $2 r$ about $x$ is contained in $U$. Suppose that $C$ is a positive number satisfying

$$
\rho(z, N \cap S) \leqslant C \rho(z, N) \text { for all } z \in S \cap U .
$$


Then

$$
\rho(y, N \cap S) \leqslant(C+1) \rho(y, S) \text { for all } y \in N \cap V .
$$

Proof. Given $y \in N \cap V$, let $z$ be a point in $S$ at minimal distance from $y$. Since $x \in S$ and $\rho(y, x) \leqslant r$, we have $\rho(z, y) \leqslant r$ and hence $z \in U$. Therefore

$$
\begin{aligned}
\rho(y, N \cap S) & \leqslant \rho(y, z)+\rho(z, N \cap S) \leqslant \rho(y, z)+C \rho(z, N) \\
& \leqslant \rho(y, z)+C \rho(z, y)=(C+1) \rho(y, z) \\
& =(C+1) \rho(y, S) . \quad \text { Q.E.D. }
\end{aligned}
$$

Proof of The Theorem. The two inequalities are equivalent by Lemma 3 , so we prove only the first one. Since the problem is nontrivial only when the given compact set $K \subset S$ intersects $N$, we may reduce easily to a local problem near a point of $N \cap S$. By applying a diffeomorphism which flattens $N$ (locally), we can reduce further to the case $N=\mathbf{R}^{n} \times\left\{O_{m-n}\right\} \subset \mathbf{R}^{n} \times \mathbf{R}^{m-n}=\mathbf{R}^{m}$. (Here $O_{p}$ denotes the origin of $\mathbf{R}^{p}$.) We may assume that $n<m$ (the case $n=m$ being trivial) and that $O_{m} \in N \cap S$. Since $K$ is compact, it suffices to find a neighborhood $V$ of $O_{m}$ in $\mathbf{R}^{m}$ for which the ratios $\rho(z, N) / \rho(z, N \cap S), z \in(V \cap S) \backslash N$, are bounded below by some positive constant.

Using Lemma 2 and the local finiteness of the collection $\left\{S_{k}\right\}$ of strata, choose a neighborhood $U$ of $O_{m}$ in $\mathbf{R}^{m}$ and an angle $\theta_{0}>0$ for which $T_{z} S_{k}$ and $T_{0} N$ are transversal and $\theta\left(T_{z} S_{k}, T_{0} N\right) \geqslant \theta_{0}$ for all strata $S_{k}$ of dimension $<m$ and all points $z \in S_{k} \cap U$. We shall show that the ratios $\rho(z, N) / \rho(z, N \cap S), z \in V \cap S \backslash N$, are bounded below by $\sin \theta_{0}$ for some set $V$ to be defined soon.

Fix the stratum $S_{k}$. For $z \in U \cap S_{k}$, regard $T_{z}=($ def $) T_{z} S_{k}$ and $T_{0} N$ as affine subspaces of $\mathbf{R}^{m}$. Thus $T_{0} N$ is identified with $N=\mathbf{R}^{n} \times\{0\} \subset \mathbf{R}^{m}$. By Lemma 1, $T_{z} \cap N$ is not empty. Let $w(z)$ be the image of $z$ under the orthogonal projection $\Pi_{z}$ of $\mathbf{R}^{m}$ onto $T_{z} \cap N$ (see Figure 1). An easy argument using the constancy of $\operatorname{dim}\left(T_{z} \cap N\right)$ as $z$ varies over $U \cap S_{k}$ shows that $w(z)$ depends continuously on $z \in U \cap S_{k}$. Let $\bar{W}_{z}$ be the vector from $z$ to $w(z)$. Then $\bar{W}$ is a continuous vector field on $U \cap S_{k}$. Let $y(z)=\Pi_{N}(z)$, the orthogonal projection of $z$ on $N$. Since $\Pi_{z}=\Pi_{z} \Pi_{N}, w(z)=\Pi_{z}(y(z))$.

Claim. For all points $z \in(S \cap U) \backslash N$,

$$
\sin \theta_{0} \leqslant \rho(z, N) /\left|\overline{W_{z}}\right| \leqslant 1 .
$$

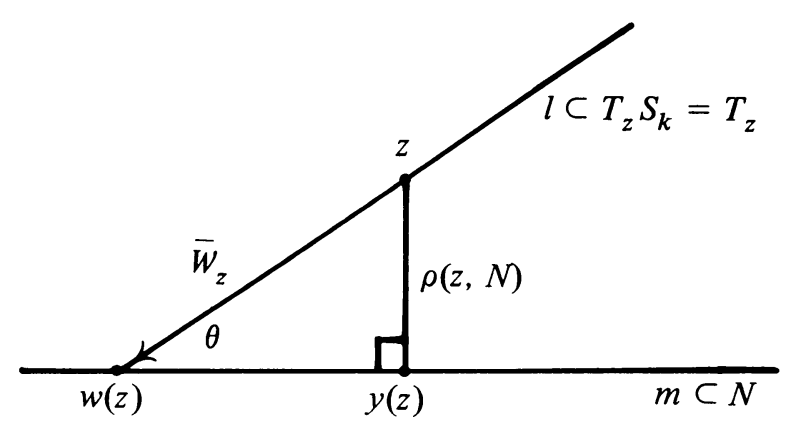

Figure 1 
Proof. Since $z \notin N, z \neq y(z)$ and $z \neq w(z)$.

Case 1. If $w(z) \neq y(z)$, then (see Figure 1) the lines $l=z w \subset T_{z}$ and $m=w y \subset N$ are both perpendicular to $T_{z} \cap N$. Therefore, $\theta(l, m) \geqslant \theta\left(T_{z}, N\right) \geqslant \theta_{0}$. Since $z y$ is perpendicular to $N$ and hence $\rho(z, y)=\rho(z, N)$, we get

$$
0<\sin \theta_{0} \leqslant \sin \theta(l, m)=\rho(z, N) /\left|\overline{W_{z}}\right| \leqslant 1 .
$$

Case 2. If $w(z)=y(z)$, then $\rho(z, N)=\left|\overline{W_{z}}\right|$, so that $\sin \theta_{0} \leqslant \rho(z, N) /\left|\bar{W}_{z}\right|=1$.

Note, in particular, that Case 2 always holds when $\operatorname{dim} S_{k}=m$.

Now choose any $z_{0} \in S_{k} \cap U$, let $z=q(t)$ be a maximal integral curve of the vector field $\bar{W}$ on $S_{k} \cap U$ with $q(0)=z_{0}$. (It exists and is $C^{1}$ [Hur, Chapter I, part A] but need not be unique, since $\bar{W}$ is only continuous.) Consider the function $f(t)=\rho(q(t), N)$. Let $s(t)$ denote the arclength along $q$ from $q(0)$ to $q(t)$. Clearly (using similar triangles and Figure 1)

$$
-d f / d s=\rho(z, N) /|\bar{W}|=\sin \theta \geqslant \sin \theta_{0}
$$

along $q$. Hence for any interval $[0, t]$ in the domain of $q$,

$$
f(0)-f(t) \geqslant\left(\sin \theta_{0}\right) s(t) \geqslant\left(\sin \theta_{0}\right) \rho\left(z_{0}, q(t)\right) .
$$

Consider now what can happen at the "end" of $q$. Thus assume that $q$ is defined on $\left[0, t_{1}\right)$ but that for all $t_{2}>t_{1}, q$ cannot be extended to an integral curve on $\left[0, t_{2}\right)$. Since $\left|W_{z}\right| \leqslant \rho(z, N) / \sin \theta_{0}$ and $\rho(z, N)$ decreases as $t$ increases, $\left|\bar{W}_{z}\right|=\left|q^{\prime}(t)\right|$ is bounded as $t \rightarrow t_{1}$. Therefore, $q(t)$ converges to some point $z_{1} \in \operatorname{Cl}\left(S_{k} \cap U\right) \subset S \cap$ $\mathrm{Cl}(U)$ as $t \rightarrow t_{1}$. We cannot have $z_{1} \in S_{k} \cap U \backslash N$, for then $\bar{W}$ would be nonzero at $z_{1}$ and hence $q$ could be extended through $t=t_{1}$, contradicting its maximality. By continuity in $(*)$,

$$
\rho\left(z_{0}, N\right)-\rho\left(z_{1}, N\right) \geqslant\left(\sin \theta_{0}\right) \rho\left(z_{0}, z_{1}\right) .
$$

Hence $\rho\left(z_{0}, z_{1}\right) \leqslant \rho\left(z_{0}, N\right) \operatorname{cosec} \theta_{0}$. Let $V$ be the closed ball of radius $r$ about 0 in $\mathbf{R}^{n}$ where $r$ is chosen so that a ball of radius $r\left(1+\operatorname{cosec} \theta_{0}\right)$ about 0 is contained in $U$. If $z_{0} \in V$, then $z_{1} \in U$. Assuming that $z_{0} \in V$, we are left with only two possibilities.

Case A. $z_{1} \in N$, hence $z_{1} \in N \cap S$. Then $\rho\left(z_{1}, N\right)=0$, so that $\rho\left(z_{0}, N\right) \geqslant$ $\left(\sin \theta_{0}\right) \rho\left(z_{0}, z_{1}\right) \geqslant\left(\sin \theta_{0}\right) \rho\left(z_{0}, N \cap S\right)$, as was to be proved.

Case B. $z_{1} \notin N$ and $z_{1} \in U \cap\left(\mathrm{Cl}\left(S_{k}\right) \backslash S_{k}\right)$. Hence $z_{1} \in U \cap S_{k_{1}}$ for some stra$\operatorname{tum} S_{k_{1}}$.

If we are in Case $\mathrm{B}$, we can repeat the previous construction, taking $z_{1}$ as the initial point of a maximal integral curve of $\bar{W}$ on $S_{k_{1}} \cap U$ which ends at some point $z_{2}$. We can concatenate the two integral curves to obtain a piecewise $C^{1}$ curve in $U \cap S$ from $z_{0}$ to $z_{2}$ which is tangent to $\bar{W}$ at all points. More generally, consider the family of all piecewise $C^{1}$ curves $q$ in $\mathbf{R}^{m}$ which lie in $U \cap S$, satisfy $q^{\prime}(t)=\bar{W}_{q(t)}$ at all $t \in$ domain $q$ and have $q(0)=z_{0}$. Using Zorn's Lemma, choose a curve $q$ in this family which is maximal in the usual sense for integral curves. The previous discussion applies verbatim to the new curve $q$. But now we cannot be in Case B, for then $q$ could be continued, contradicting its maximality. Hence, $q$ ends at a point of $N \cap S$ (Case A) and we are done. Q.E.D. 
REMARK. Zorn's Lemma was necessary because the stratification is only (a)-regular. For (b)-regular stratifications, each piece of the constructed integral curve would end in a stratum of strictly lower dimension, forcing the process to end after a finite number of steps. For (a)-regular stratifications these dimensions are only nonincreasing.

Corollary to Proof of Theorem. Let $(S, \Sigma)$ be a closed (a)-regular stratified subset of $\mathbf{R}^{m}$. Let $n$ be a nonnegative integer $<m$. For each $y \in \mathbf{R}^{m-n}$ let $N_{y}=\mathbf{R}^{n} \times$ $\{y\} \subset \mathbf{R}^{n} \times \mathbf{R}^{m-n}=\mathbf{R}^{m}$. Assume that $0 \in S$ and that $N=(\mathrm{def}) N_{0}$ is transversal to $(S, \Sigma)$. Then there exist a positive constant $C$ and neighborhoods $V_{1}$ of 0 in $N$ and $V_{2}$ of 0 in $\mathbf{R}^{m-n}$ such that

$$
\rho((x, y), S) \geqslant C \rho\left((x, y), N_{y} \cap S\right) \text { for all } x \in V_{1} \text { and all } y \in V_{2} .
$$

Proof. Let $U$ be as in the proof of the Theorem. Observe that since the $N_{y}$ are parallel affine subspaces of $\mathbf{R}^{m}, T_{(0, y)} N_{y}=T_{0} N_{0}=\mathbf{R}^{n} \times\{0\}$ for all $y$. Therefore, the proof of the Theorem goes through for $N_{y}$ in place of $N$, provided that the radius $r$ of the ball $V$ about $0 \in \mathbf{R}^{m}$ is now chosen so that a ball of radius $r\left(1+2 \csc \theta_{0}\right)$ about 0 is contained in $U$. (This is necessary since if $V$ intersects $N_{y}$, then points of $V$ lie at distance $\leqslant 2 r$ from $N_{y}$ (but $\leqslant r$ from $N_{0}$ ).) Hence for all points $z \in V \cap S$ and all $y$ for which $V$ intersects $N_{y}$,

$$
\rho\left(z, N_{y} \cap S\right) \leqslant\left(\operatorname{cosec} \theta_{0}\right) \rho\left(z, N_{y}\right) .
$$

Let $V^{\prime}$ be the ball of radius $r / 2$ about $0 \in \mathbf{R}^{m}$. Since $0 \in S$, Lemma 3 implies that for all $y \in \mathbf{R}^{m-n}$ and all $w \in V^{\prime} \cap N_{y}$

$$
\rho\left(w, N_{y} \cap S\right) \leqslant\left(1+\operatorname{cosec} \theta_{0}\right) \rho(w, S) .
$$

We finish by choosing open sets $V_{1}$ and $V_{2}$ so that $0 \in V_{1} \times V_{2} \subset V^{\prime}$ and setting $C=1 /\left(1+\operatorname{cosec} \theta_{0}\right)$. Q.E.D.

Proof of Corollary 1. Factor the map $f: \mathbf{R}^{n} \rightarrow \mathbf{R}^{m}$ as $f_{3} f_{2} f_{1}$, where $f_{1}$ is the diffeomorphism $x \mapsto(x, f(x))$ of $\mathbf{R}^{n}$ onto graph $f$, regarded as a submanifold of $\mathbf{R}^{n+m}, f_{2}$ is the inclusion of graph $f$ into $\mathbf{R}^{n} \times \mathbf{R}^{m}$ and $f_{3}$ is the projection $\mathbf{R}^{n} \times \mathbf{R}^{m} \rightarrow$ $\mathbf{R}^{m}$. Let $\sim$ denote the equivalence relation "bounded by constant multiples of each other". Since $f_{1}$ is a diffeomorphism,

$$
\rho_{n}\left(x, f^{-1}(S)\right) \sim \rho_{n+m}\left((x, f(x)), f_{1}\left(f^{-1}(S)\right)\right) \quad \text { for } x \in K .
$$

Now $f_{1}\left(f^{-1}(S)\right)=f_{2}^{-1} f_{3}^{-1}(S)=\left(\mathbf{R}^{n} \times S\right) \cap$ graph $f$. The transversality of $f$ to $(S, \Sigma)$ implies that graph $f$ is transversal to $\mathbf{R}^{n} \times S$ stratified by $\left\{\mathbf{R}^{n} \times S_{i}\right\}$, where $\left\{S_{i}\right\}$ are the strata of $(S, \Sigma)$. Hence by the Theorem,

$$
\rho_{n+m}\left((x, f(x)), f_{1}\left(f^{-1}(S)\right)\right) \sim \rho_{n+m}\left((x, f(x)), \mathbf{R}^{n} \times S\right) \text { for } x \in K .
$$

But $\rho_{n+m}\left((x, f(x)), \mathbf{R}^{n} \times S\right)=\rho_{m}(f(x), S)$. Q.E.D.

Lemma 4. Let $U$ be open in $\mathbf{R}^{n}$, and let $f$ and $f_{i}, i=1,2, \ldots$, be $C^{k}$ functions $(0 \leqslant k<\infty)$ from $\mathbf{R}^{n}$ to $\mathbf{R}^{m}$. Assume that $D^{\alpha} f_{i}$ converges to $D^{\alpha} f$ uniformly on $U$ for each partial derivative $D^{\alpha}$ of order $|\alpha| \leqslant k$. Then there exists a $C^{k}$ function $F$ : $U \times \mathbf{R} \rightarrow \mathbf{R}^{m}$ such that the sequence of functions $F_{1 / j}(x)$ (where $F_{t}(x)=F(x, t)$ ) is a subsequence of $\left\{f_{i}\right\}$. (It follows automatically that $F_{0}=f$.) 
PROoF (compare proof of Lemma 4.2 on p. 146 of [GWPL]). Let $g_{j}=f_{j}-f$. For any $C^{k}$ function $g: U \rightarrow \mathbf{R}^{m}$, resp. $U \times \mathbf{R} \rightarrow \mathbf{R}^{m}$, let $\|g\|_{k}$ be the supremum of $\left|\left(D^{\alpha} g\right)(x)\right|$ over all $\alpha$ with $|\alpha| \leqslant k$ and all $x \in U$, resp. $U \times \mathbf{R}$. By replacing $\left\{f_{j}\right\}$ by a subsequence, we may assume that $\left\|g_{j}\right\|_{k}<j^{-2(k+1)}$ for all $j$. Choose a $C^{k}$ function $h$ : $\mathbf{R} \rightarrow \mathbf{R}$ with $h(0)=1$ and $h(t)=0$ when $|t| \geqslant 1$. Define

$$
F(x, t)=f(x)+\sum_{j=1}^{\infty} h\left(2 j^{2}(t-1 / j)\right) g_{j}(x) .
$$

One verifies easily that $F(x, 1 / r)=f_{r}(x)$, and that the partial sums $F^{s}(x, t)=f(x)$ $+\sum_{j=1}^{s} h\left(2 j^{2}(t-1 / j)\right) g_{j}(x)$ form a Cauchy sequence in the \|\|$_{k}$ norm. Hence $F(x, t)$ is a well-defined $C^{k}$ function on $U \times \mathbf{R}$. Q.E.D.

Proof of Corollary 2. In the contrary case one could find a sequence of $C^{1}$ functions $f_{q}: U \rightarrow \mathbf{R}^{m}$ and a sequence of points $x_{q} \in K$ such that $f_{q} \rightarrow f$ and $d f_{q} \rightarrow d f$ uniformly on $U$ for which

$$
\rho_{n}\left(x_{q}, f_{q}^{-1}(S)\right) \times \rho_{m}\left(f_{q}\left(x_{q}\right), S\right),
$$

where $\sim$ is as in the proof of Corollary 1 . Without loss of generality we may assume that $\left\{x_{q}\right\}$ converges to some point $x \in K$. If $f(x) \notin S$, then we could find a small ball $B$ about $x$, an integer $Q$, and a number $\varepsilon>0$ such that $\rho(f(B), S)>\varepsilon$ and $\rho\left(f_{q}(B), S\right)>\varepsilon$ for all $q>Q$. Let $B^{\prime}$ be a ball about $x$ of smaller radius than $B$ and choose $Q^{\prime}>Q$ co that $x_{q} \in B^{\prime}$ for $q>Q^{\prime}$. Then both sides of (1) are bounded below (and, of course, above) by positive numbers for $q>Q^{\prime}$, contradicting (1). Hence $f(x) \in S$.

By Lemma 4 we may find a $C^{1}$ function $F: U \times \mathbf{R} \rightarrow \mathbf{R}^{m}$ such that $F_{1 / j}(x)$ is a subsequence of $\left\{f_{q}\right\}$ (here $F_{t}(x)=F(x, t)$ ).

By a result of E. A. Feldman [Fel, Tro], the set of $C^{1}$ maps from $U$ to $\mathbf{R}^{m}$ which are transversal to $(S, \Sigma)$ is open in the Whitney $C^{1}$ topology. Let $V$ be an open neighborhood of $x$ whose closure is compact and contained in $U$. Since $F_{0}=f$ is transversal to $(S, \Sigma)$, we can find an open interval $J$ containing 0 such that $F_{t} \mid V$ is transversal to $(S, \Sigma)$ for all $t \in J$. It follows that $F \mid V \times J$ is also transversal to $(S, \Sigma)$. By [GWPL, p. 13], now, $F^{-1} S \cap V \times J$ is an (a)-regular stratified set with strata $F^{-1} S_{i} \cap V \times J\left(\left\{S_{i}\right\}=\right.$ strata of $\left.(S, \Sigma)\right)$. Also, $V \times\{t\}$ is transversal to $F^{-1} S$ for each $t \in J$.

Now apply the corollary to the proof of the Theorem, together with a localization argument, to $F^{-1} S \cap V \times J$. We find thereby compact neighborhoods $V_{1}$ of $x$ in $V$ and $V_{2}$ of 0 in $J$ such that

$$
\rho\left((v, t), F^{-1} S \cap V \times J\right) \sim \rho\left((v, t), F^{-1} S \cap V \times\{t\}\right) \quad \text { for }(v, t) \in V_{1} \times V_{2} .
$$

Next, apply Corollary 1 to $F: V \times J \rightarrow \mathbf{R}^{m}$, with $\mathrm{Cl}\left(V_{1} \times V_{2}\right)$ for $K$, to get

$$
\rho\left((v, t), F^{-1}(S) \cap V \times J\right) \sim \rho(F(v, t), S) \text { for }(v, t) \in \mathrm{Cl}\left(V_{1} \times V_{2}\right) .
$$

From (2), (3) and the identity $\rho\left((v, t), F^{-1}(S) \cap V \times\{t\}\right)=\rho\left(v, F_{t}^{-1}(S) \cap V\right)$ we get

$$
\rho_{n}\left(v, F_{t}^{-1}(S) \cap V\right) \sim \rho_{m}\left(F_{t}(v), S\right) \quad \text { for }(v, t) \in V_{1} \times V_{2} .
$$


In particular, we find that

$$
\rho_{n}\left(x_{q}, f_{q}^{-1}(S) \cap V\right) \sim \rho_{m}\left(f_{q}\left(x_{q}\right), S\right) \text { for large enough } q .
$$

But for large enough $q$, the left sides of (1) and (5) are equal. Hence (1) and (5) are contradictory. Q.E.D.

\section{REFERENCES}

[Fel] E. A. Feldman, The geometry of immersions. I, Trans. Amer. Math. Soc. 120 (1965), 185-224.

[GG] M. Golubitsky and V. Guillemin, Stable mappings and their singularities, Springer-Verlag. New York, 1973, 1980.

[GWPL] C. G. Gibson et al., Topological stability of smooth mappings, Lecture Notes in Math., vol. 552. Springer-Verlag, New York, 1976.

[Hur] W. Hurewicz, Lectures on ordinary differential equations, M. I. T. Press, 1958.

[Kuo] T.-C. Kuo, The ratio test for analytic Whitney stratifications, Liverpool Singularities Symposium I, Lecture Notes in Math., vol. 192, Springer-Verlag, New York, 1971, pp. 141-149.

[Loj] S. Lojasiewicz, Sur le probleme de la division, Studia Math. 18 (1959), 87-136.

[Mal] B. Malgrange, Ideals of differentiable functions, Oxford Univ. Press, London, 1966.

[MS] M. Mostow and S. Shnider, Joint continuity of division of smooth functions. I: Uniform Lojasiewicz estimates, Trans. Amer. Math. Soc. 292 (1985), 573-583.

[Tro] D. J. A. Trotman, Stability of transversality to a stratification implies Whitney (a)-regularity, Invent. Math. 50 (1979), 273-277.

[Wh1] H. Whitney, Local properties of analytic varieties in differential and combinatorial topology' (S. S. Cairns, ed.), Princeton Univ. Press, Princeton, N. J., 1965, pp. 205-244.

[Wh2] _. Tangents to an analytic variety, Ann. of Math. (2) 81 (1965), 496-549.

Department of Mathematics, North Carolina State University, Raleigh, North Carolina 27650

Current address: Clarity, Inc., P. O. Box 3112, Herzelia 46103, Israel 